\title{
Angioedema without wheals: a clinical update
}

\author{
Okan Gülbahar
}

Division of Immunology-Allergy, Department of Internal Medicine, Ege University School of Medicine, İzmir, Turkey

\begin{abstract}
Angioedema without wheals (urticaria) represents a heterogeneous group of clinically indistinguishable diseases of hereditary or acquired etiology. Hereditary angioedema is a rare inherited condition leading to recurrent, sometimes life-threatening angioedema attacks in subcutaneous tissues and gastrointestinal and oropharyngeal mucosa dating back to childhood or adolescence. Most of these patients have mutations in the SERPING1 gene, causing either low $\mathrm{C} 1$ inhibitor production (hereditary angioedema with $\mathrm{C} 1$ inhibitor deficiency type I) or the production of dysfunctional $\mathrm{C} 1$ inhibitor (hereditary angioedema with $\mathrm{C} 1$ inhibitor deficiency type II). Hereditary angioedema with normal $\mathrm{C} 1$ inhibitor has been defined later. Although $\mathrm{C} 1$ inhibitor concentration and function are in the normal range, it leads to typical hereditary angioedema symptoms owing to mutations in FXII, PLG, $A N G P T 1, K N G 1$, and MYOF genes. Patients who exhibit none of these genetic mutations despite having a similar clinical presentation are classified as having unknown hereditary angioedema. Fewer than 1 in 10 patients with $\mathrm{C} 1$ inhibitor deficiency have acquired angioedema with $\mathrm{C} 1$ inhibitor deficiency. The clinical presentation is very similar to that of hereditary angioedema, making it difficult to distinguish these 2 conditions clinically. Unlike hereditary angioedema, there are no genetic mutations, and family history and symptoms tend to appear later in life. Acquired angioedema with $\mathrm{C} 1$ inhibitor deficiency is commonly associated with lymphoproliferative
\end{abstract}

and autoimmune diseases. Angioedema attacks might start 1 year before the underlying disease in acquired angioedema with $\mathrm{C} 1$ inhibitor deficiency. Approximately half of the patients admitted to the hospital for acute angioedema are patients receiving angiotensin-converting enzyme (ACE) inhibitor therapy. Angioedema typically occurs on the lips, tongue, mouth, pharynx, and subglottic regions. Patients may require hospitalization and intensive care monitoring owing to airway involvement. Idiopathic histaminergic acquired angioedema may be diagnosed only when any possible causes of histaminergic angioedema are excluded (foods, drugs, animal dander, aeroallergens, insect stings, latex, and others), and the symptoms respond well to antihistamine treatment. Idiopathic nonhistaminergic acquired angioedema should be considered when all other types of recurrent angioedema have been ruled out and patients do not respond to high-dose antihistamines. The lack of a standard biochemical laboratory test for patients with idiopathic histaminergic acquired angioedema, idiopathic nonhistaminergic acquired angioedema, angiotensin-converting enzyme inhibitor-induced acquired angioedema, and hereditary angioedema with normal $\mathrm{C} 1$ inhibitor makes the diagnosis more challenging. Future efforts should focus on increasing awareness of all the rare types of angioedema among physicians and developing more straightforward and more accessible diagnostic methods.
Angioedema is a transient, nonpitting, localized swelling of the subcutaneous and mucosal tissues. ${ }^{1,2}$ Unlike urticaria, which involves the superficial layers of the skin, this swelling occurs in the lower layers of the dermis and subcutaneous tissues as a result of fluid extravasation into the interstitial spaces, and it generally develops suddenly. Angioedema tends to be asymmetrical and can occur anywhere on the body, although the most frequently affected areas are the face, lips, mouth, throat, larynx, extremities, genital area, and the gastrointestinal tract. ${ }^{1,2}$ The skin in the affected area is usually normal in color but can sometimes be erythematous. There is usually no itching. Pain and burning sensation may be present at varying rates. Angioedema varies in severity and is often self-limited to periods ranging from a few hours to several days. ${ }^{1}$

Many different conditions can cause recurrent angioedema, and their underlying mechanisms are hard to be distinguished solely on the basis of the patient's clinical presentation..$^{2-6}$ Angioedema is the most common cause of hospital admissions among all allergic diseases after asthma. ${ }^{7}$ More than a third of patients with recurrent angioedema also have urticaria. ${ }^{3-5,8}$ In such cases, diagnosis and treatment with standard approaches are generally more straightforward. However, in some patients, recurrent angioedema attacks are not accompanied by urticaria. In these cases, isolated angioedema may either occur owing to mast cell activation (histaminergic angioedema) or through mediators, such as bradykinin (nonhistaminergic angioedema).

The various subtypes of angioedema can be determined through a detailed clinical history, physical examination, and laboratory tests. Clarifying the underlying mechanism is crucial to guiding acute treatment and preventing subsequent attacks, screening family members if necessary, and advising the patient to prevent recurrence of attacks. However, in about one-third of patients who pres-

Address for Correspondence: Okan Gülbahar, Division of Immunology-Allergy, Department of Internal Medicine, Ege University School of Medicine, İzmir, Turkey

e-mail: okan.gulbahar@ege.edu.tr

Received: November 7, 2020 Accepted: January 25, 2021 •DOI: 10.5152/balkanmedj.2021.20060

Available at www.balkanmedicaljournal.org

ORCID iD of the author: O.G. 0000-0002-5341-9070.

Cite this article as:

Gülbahar O, Angioedema without wheals: a clinical update. Balkan Med J. 2021;38(2):73-81.

Copyright@Author(s) - Available online at http://balkanmedicaljournal.org/ 
ent to the hospital owing to angioedema, no cause can be identified, and they are diagnosed as having idiopathic angioedema. ${ }^{9,10}$ In daily

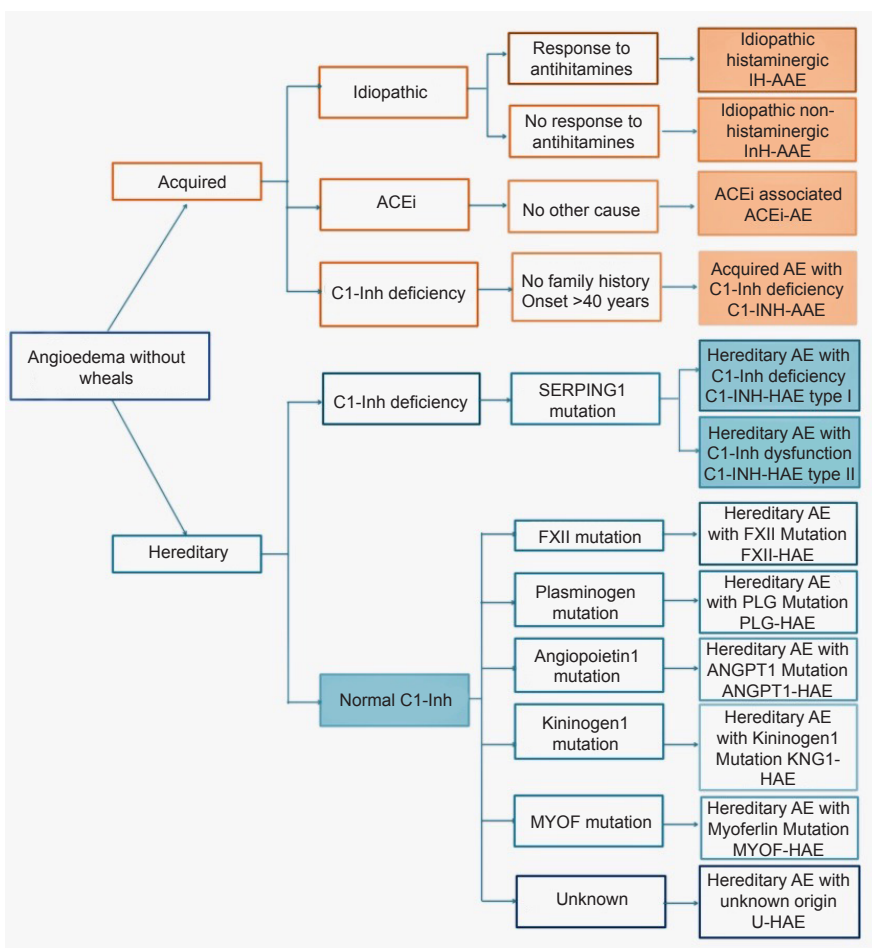

FIG. 1. Classification of angioedema without urticaria (modified from reference 15).

IH-AAE, Idiopathic histaminergic acquired angioedema; InH-AAE, Idiopathic non-histaminergic acquired angioedema; ACEi-AE, ACEi associated angioedema; C1-INH-AAE, Acquired AE with C1-Inh deficiency; C1-INH-HAE type I, Hereditary AE with C1-Inh deficiency; C1-INH-HAE type II, Hereditary AE with C1-Inh dysfunction; FXII-HAE, Hereditary AE with FXII Mutation; PLG-HAE, Hereditary AE with PLG Mutation; ANGPT1-HAE, Hereditary AE with ANGPT1 Mutation; KNG1HAE, Hereditary AE with Kininogen1 Mutation; MYOF-HAE, Hereditary AE with Myoferlin Mutation; U-HAE: Hereditary AE with unknown origin. practice, the most commonly encountered angioedema is histaminergic angioedema. ${ }^{6}$ Of all hospital admissions, the most common cause of angioedema is drugs, especially angiotensin-converting enzyme inhibitors (ACEi), nonsteroidal anti-inflammatory drugs (NSAIDs), and beta-lactam antibiotics. ${ }^{9-13}$

Recently, angioedema without wheals has been evaluated under 2 main categories: hereditary angioedema (HAE) and acquired angioedema (AAE) (Figure 1). In this article, 7 clinical conditions, 3 of which are hereditary and 4 of which are acquired, are summarized. ${ }^{14,15}$

\section{HEREDITARY ANGIOEDEMA}

HAE is a rare and inherited condition characterized by recurrent, noninflammatory, nonpitting, self-limiting swellings affecting subcutaneous tissues and gastrointestinal and oropharyngeal mucosa without urticaria. ${ }^{16-20}$ There are 2 types of HAE: HAE with $\mathrm{C} 1$ inhibitor (C1-INH) deficiency (C1-INH-HAE) and HAE with normal C1-INH (nC1-INH-HAE). The clinical characteristics that follow are generally known for C1-INH-HAE. HAE's minimal prevalence in the population is 1.41 in 100,000 persons and ranges from 1 in 10,000 persons to 1 in 50,000 persons. ${ }^{20-22}$ HAE shows no ethnic- or sex-based differences but tends to be more severe in women. The angioedema attacks are usually disfiguring, potentially causing severe morbidity and might be life threatening. Attacks vary in frequency and severity and are difficult to predict, although prodromal symptoms may precede. They generally progress over 24 hours and resolve within 2-5 days. Some members of the family often have similar symptoms. HAE is often confused with other causes of angioedema, including allergic angioedema; however, unlike histaminergic angioedema, which typically starts to swell in minutes and resolves in hours, swellings develop more slowly; do not respond well to treatment with corticosteroids, antihistamines, or adrenaline; and resolve in days (Table 1). This confusion can sometimes lead to a delay in delivering appropriate treatment, re-

TABLE 1. Clinical clues based on clinical features

\begin{tabular}{|c|c|c|}
\hline Clinical features & Bradykinin mediated & Histamine mediated \\
\hline $\begin{array}{l}\text { Possible agents (foods, drugs, insects, animal dander, } \\
\text { aeroallergens, and others) }\end{array}$ & $\begin{array}{l}\text { ACEI, DPP-IV inhibitors, NEP inhibitors, } \\
\text { estrogens for HAE }\end{array}$ & May be present \\
\hline Age of onset & $\begin{array}{c}\text { 3-20 years for } \mathrm{HAE} \\
>40 \text { years for } \mathrm{C} 1 \text {-INH-AAE }\end{array}$ & Any age \\
\hline Family history & $\begin{array}{l}\text { Present in }>75 \% \text { for } \mathrm{HAE} \\
\text { None for AAE }\end{array}$ & Rare \\
\hline Swelling & $\begin{array}{l}\text { Develops slowly (hours), recovers slowly } \\
\text { (48-72 hours or more) }\end{array}$ & $\begin{array}{l}\text { Quick onset (minutes), recovers faster } \\
\qquad(12-24 \text { hours })\end{array}$ \\
\hline Severity & More severe & Less severe \\
\hline Urticaria & Absent & Often \\
\hline Risk of recurrent laryngeal edema & Significant & Low \\
\hline Recurrent abdominal attacks & Frequent & Rare \\
\hline Prodromal symptoms & May precede (frequent in HAE) & None \\
\hline $\begin{array}{l}\text { Response to antihistamines, corticosteroids, and adren- } \\
\text { alin }\end{array}$ & Poor & Excellent \\
\hline
\end{tabular}


sulting in severe consequences and even death. The swelling does not have well-defined borders, is usually not painful but bothersome, and develops owing to plasma leakage from postcapillary venules into the dermal layers of the skin. This leakage is mainly associated with the absence or dysfunction of a protein called $\mathrm{C} 1$ INH owing to a mutation in the SERPING1 gene. ${ }^{18,19}$ The increase in vascular permeability that causes angioedema in HAE is related to the mediators of the contact system or kallikrein-kinin pathway. C1-INH regulates the contact system through the inhibition of plasma kallikrein and coagulation factor FXIIa. This inhibition suppresses the formation of bradykinin. Bradykinin is a nonapeptide produced as a result of contact system activation. It binds to Bradykinin 2 receptors (B2R) on vascular endothelial cells, leading to increased vascular permeability and edema. Bradykinin concentrations increase acutely in patients with HAE. ${ }^{23}$

\section{Disease Onset}

In most patients, recurrent swelling attacks begin in childhood. Subcutaneous swellings and angioedema attacks with abdominal involvement generally emerge during this period; however, they are relatively mild, and the diagnosis is often overlooked. Disease severity has a tendency to increase in both men and women during puberty. ${ }^{20}$ The frequency and severity of attacks can vary significantly among patients and within the same patient over time. ${ }^{21}$ The disease tends to be more severe in patients with an early onset of symptoms compared with those with later onset. Without treatment, patients may experience an angioedema attack every 1-2 weeks on average. In some cases, episodes can occur more frequently (e.g., once a week) or less frequently (e.g., once a year). ${ }^{22,24}$

\section{Triggering Factors}

Various factors can trigger HAE attacks. These factors include stress, pressure, trauma, dental and some medical interventions (such as endoscopy, bronchoscopy, colonoscopy, intubation, and surgical procedures), infections, and hormonal changes. ${ }^{25}$ However, attacks can also begin with no apparent cause in many patients. A substantial proportion of patients are aware of certain factors that trigger attacks. Menstruation is a primary trigger; some women may be completely asymptomatic apart from this period. In particular, estrogen-containing drugs and ACE inhibitors (ACEi) can trigger attacks, and these drugs should be avoided in patients with HAE. ${ }^{26,27}$ In some women, discontinuing the use of estrogen-containing oral contraceptives alone can be enough to prevent further attacks. Patients whose symptoms are more severe during their menstrual period tend to experience more severe symptoms during pregnancy. ${ }^{28,29}$

\section{Prodromal Symptoms}

Some patients describe various signs and symptoms before the onset of attacks. ${ }^{25}$ In some cases, episodes of abdominal pain may be accompanied by nausea and vomiting. There may be a tingling sensation in the area 1-24 hours before the swelling appears. Erythema marginatum, which sometimes may be misinterpreted as urticaria, occurs in one-third of the patients. These pink, macular rings do not itch and usually occur on the trunk's inner surfaces, arms, and legs but not on the face. Asthenia, anxiety, itching, and muscle pain are other prodromal symptoms that may precede attacks. ${ }^{18-20}$

\section{Affected Areas}

HAE attacks can occur in the face, extremities, genitals, respiratory tract, and intestinal wall. Most attacks affect 1 or 2 areas. Angioedema involving multiple sites simultaneously is seen in only $15-30 \%$ of cases. ${ }^{30}$ About $50 \%$ of the swellings occur in the subcutaneous area, and $48 \%$ occur in the gastrointestinal tract. Angioedema attacks with the gastrointestinal tract, larynx, uvula, and tongue involvement are reported to be $97 \%, 57 \%, 22 \%$, and $9 \%$, respectively. ${ }^{22}$ On an attack basis, $45 \%$ of subcutaneous swellings occur in the extremities, $2 \%$ in the genital area, $1.6 \%$ in the face, and $0.9 \%$ in the trunk and neck. ${ }^{22}$

Similar edema often occurs in the intestinal wall and causes severe spasmodic abdominal pain and bloating. A total of $97 \%$ of patients describe gastrointestinal angioedema at some point in their lives, and abdominal attacks accompany about $50 \%$ of all HAE episodes. ${ }^{23} \mathrm{In}$ some cases, the episodes of abdominal pain may be the most common symptom. The abdominal pain may be very severe and may be mistakenly considered a surgical emergency. Therefore, 1 of 3 patients has a history of unnecessary surgical intervention. ${ }^{25}$ The fluid that leaks from the bowel loops can sometimes cause ascites and hypotension. Diarrhea may occur during the remission of abdominal attacks. An essential point concerning abdominal attacks is that they do not necessarily occur together with peripheral attacks. Although rare, some patients may have only gastrointestinal symptoms, making the diagnosis more difficult and further delaying an accurate diagnosis in these patients. ${ }^{31}$ Patients can experience such attacks once a month or 2-3 times a year and may also have been diagnosed erroneously with Familial Mediterranean fever. ${ }^{32,33}$

More than $50 \%$ of patients with HAE experience at least 1 episode of laryngeal edema during their lifetime. ${ }^{25}$ Laryngeal symptoms usually occur between the ages of 11 and 45 years and are rare before the age of 3 years. ${ }^{34,35}$ The time between onset and maximum development of laryngeal edema is about 8 hours. ${ }^{34,35}$ Although laryngeal edema occurs in $<1 \%$ of attacks, it can be fatal. ${ }^{9}$ Before the current treatments for HAE were available, it was reported that 1 in 3 family members with untreated HAE had a history of asphyxia. The mortality rate can be up to $50 \%$ in undiagnosed HAE. Laryngeal edema develops within hours in most HAE cases, but airway obstruction occurring in $<1$ hour has also been reported. ${ }^{9}$

Patients with HAE are generally healthy outside episodes of angioedema attacks. ${ }^{32}$ However, the disease has a severe impact on patients' daily lives and can be unpredictable or even life threatening. Many have relatives who died owing to asphyxia. Attacks involving the upper respiratory tract are associated with high mortality, and patients live with the constant threat of laryngeal edema. ${ }^{36,37}$ Swelling of the gastrointestinal mucosa mimics surgical emergencies owing to common features, such as nausea, vomiting, and severe abdominal pain. ${ }^{36,37}$ During attacks, patients are rendered partially or entirely unable to carry out their daily activities. Even attacks in the extremities can cause significant limitations. A total of $100 \%$ of patients state that their disease prevents them from being more successful at school or work, and 50\% were unable to go to school or work owing to their previous attack; attacks are believed to cause 20-100 days of lost capacity per year, and uncertainty places a severe psychological burden on patients. ${ }^{32}$ 


\section{Types of Hereditary Angioedema}

\section{Hereditary Angioedema with C1 Inhibitor Deficiency}

C1-INH-HAE (OMIM\#106100) occurs owing to mutations in the SERPING1 gene, which encodes C1-INH. These mutations cause either low C1-INH production (C1-INH-HAE type I, $85 \%$ of cases) or the production of dysfunctional C1-INH (C1-INH-HAE type II, $15 \%$ of cases). Over 700 mutations that cause C1-INH-HAE have been identified in this region to date. ${ }^{38,39} \mathrm{C} 1$-INH-HAE shows autosomal dominant inheritance and does not skip generations. De novo mutations are detected in $6-25 \%$ of patients. ${ }^{39}$ In other words, the mutation originates in that person. Therefore, the absence of family history does not rule out this disease. About $5 \%$ of patients may be asymptomatic despite carrying a mutation. ${ }^{20}$ Besides, $5-10 \%$ of patients with C1-INH-HAE have no detectable mutation in the SERPING1 region. It is not fully understood how C1-INH-HAE occurs in patients with no SERPING1 mutation. Factors leading to increased post-translational consumption of C1-INH might explain the disease pathogenesis in some of these patients. ${ }^{33} \mathrm{~A}$ genetic test that may skip the screening of the entire SERPING1 gene (e.g., introns) may be one possible reason why mutation could not be detected in all patients. ${ }^{39}$ In C1-INH-HAE type 1, circulating C1-INH concentration is always low, with associated low C1-INH function. However, the disease's clinical presentation is highly variable and is not usually correlated with C1-INH level and function. The frequency and severity of attacks vary among family members who carry the same mutation and even within the same individual over time.

The diagnosis of C1-INH-HAE can be made by evaluating the presence of typical recurrent bradykinin-mediated angioedema attacks, family history, and low complement components $\mathrm{C} 4$ and $\mathrm{C} 1-\mathrm{INH}$ levels and function. C1-INH levels should be evaluated with caution. The concentrations may be lower than expected in pregnant women with no disease owing to increased volume and in infants aged $<1$ year owing to insufficient production. In C1-INH-HAE type I, both $\mathrm{C} 1-\mathrm{INH}$ and $\mathrm{C} 4$ levels are below $50 \%$ of the reference level. ${ }^{40} \mathrm{C} 4$ levels are low in most patients with HAE, especially during an attack, but normal levels do not rule out the disease. ${ }^{40}$ In C1-INH-HAE type II, a nonfunctional protein is synthesized. Therefore, $\mathrm{C} 4$ concentration and $\mathrm{C} 1-\mathrm{INH}$ function are low, whereas blood C1-INH concentrations are normal or even high. ${ }^{40}$

\section{Hereditary Angioedema with Normal C1 Inhibitor}

Another type of HAE, formerly called HAE type III, was later described as C1-INH-HAE (OMIM\#610618). Although this group of patients exhibits typical symptoms of HAE, their C1-INH concentration and function are within the normal range. This condition was first described in women during pregnancy or estrogen use. ${ }^{41}$ Some affected male relatives were also reported later. ${ }^{42}$

Although there is considerable similarity in the symptoms of all the 3 types of HAE, patients with C1-INH-HAE may have some notable characteristics. The disease commonly affects women, especially those using oral contraceptives. The clinical presentation is very similar to those of C1-INH-HAE type 1 and type 2. Symptoms tend to appear in adulthood and are rare in children aged 10 years. There are more symptom-free periods than in HAE-C1-INH, and facial swelling is more common C1-INH-HAE. Recurrent tongue swelling may be the primary symptom. Although gastrointestinal attacks are reported to be less common, ${ }^{43}$ in a French cohort, patients had a greater rate of abdominal attacks than that had in C1-INH-HAE ( $80 \%$ vs $61 \%){ }^{44}$ There is currently no standard biochemical laboratory test developed to diagnose nC1-INH-HAE. Therefore, a genetic study is needed to confirm the diagnosis and identify the specific genetic mutation. ${ }^{45}$ Unfortunately, this type of genetic testing is not yet common worldwide.

HAE-specific mutations have been identified to date in some of the patients with nC1-INH-HAE, inclu ding the recently reported MYOF gene mutation (Myoferlin-HAE) ${ }^{46}$ The first of these was associated with a mutation of the coagulation factor FXII (FXII-HAE; ORPHA:100054), which causes increased bradykinin levels. ${ }^{43}$ FXII-HAE shows low penetrance. Males are rarely symptomatic, and most female patients have angioedema attacks during pregnancy or when taking estrogen-containing medications. Facial attacks are more common in patients with FXII mutation than in those with C1-INH-HAE. FXII mutations detected to date are p.Thr328Lys, p.Thr328Arg, c.892_909dup, and c.971_108fs24del72. ${ }^{47}$ Later, it was shown that this clinical presentation could also be caused by mutations detected in the PLG gene (PLG-HAE, p.Lys330Glu), ANGPT1 gene (ANGPT1-HAE, p.Ala119Ser), and KNG1 gene (KNG1-HAE, p.Met379Lys). ${ }^{48}$

Some clinical features of nC1-INH-HAE are exceptional. Erythema marginatum observed as a prodromal symptom in C1-INH-HAE has not been reported in any of the nC1-INH-HAE types to date. The triggering effects of estrogens on the disease are much more pronounced in FXII-HAE than in other types of nC1-INH-HAE. A high frequency of tongue swelling in PLG-HAE is reported as the only type of swelling that occurs more frequently than in other types of nC1-INH-HAE without any other clinical symptoms. ${ }^{48}$

Patients who exhibit none of the genetic mutations identified to date despite having a similar clinical presentation are classified as HAE-Unknown (U-HAE). It is predicted that new mutations will be detected in this group of patients soon.

\section{Unmet Needs}

The biggest problem related to HAE is low clinical awareness. ${ }^{49}$ The average time from symptom onset to diagnosis was 22 years in $1977,>10$ years in 2005, and 8.5 years in 2013. ${ }^{50}$ However, this time shortens dramatically with higher physician awareness of the disease. The mortality rate is 24 times higher in undiagnosed patients than in diagnosed ones. ${ }^{35}$ Therefore, it is vitally essential that undiagnosed patients be recognized and treated. HAE should be suspected in patients with recurrent angioedema attacks without urticaria, recurrent attacks of abdominal pain, distention and vomiting, and laryngeal edema history, especially if they have other family members with similar symptoms. ${ }^{51}$

\section{ACQUIRED ANGIOEDEMA}

\section{Idiopathic Histaminergic Acquired Angioedema}

Acquired recurrent angioedema may occur through different mechanisms. One of these is histaminergic angioedema, which develops as a result of mast cells and basophil activation. This type of angioedema is usually accompanied by urticaria and/or pruritus in 
$90 \%$ of the cases. ${ }^{3-5}$ However, urticaria may not always be present. The absence of urticaria may cause difficulties in diagnosis. Acute allergic angioedema typically occurs after exposure to allergens, such as food, drugs, latex, animal danders, or after insect stings. Angioedema is sometimes a component of anaphylaxis. Histaminergic angioedema, whether through immunoglobulin E ( $\operatorname{IgE}$ ) or direct mast cell activation, occurs owing to the release of potential vasodilators from mast cells (especially histamine), causing venular dilation and increased local circulation and endothelial permeability. Angioedema in children also usually occurs by this pathway, and typical examples of this are angioedema associated with food, drugs, and infections. ${ }^{8,9}$ These patients may also have other allergic conditions, such as atopic dermatitis, allergic rhinitis, and/or asthma. Various drugs or pharmaceuticals, opiates, and radiocontrast agents may cause angioedema (with or without urticaria) through direct mast cell activation. Skin tests or in vitro tests are generally not helpful in the diagnosis of this type of angioedema. Aspirin and NSAIDs can cause acute urticaria and/or angioedema in some people. Through cyclooxygenase (COX)1 enzyme inhibition, NSAIDs increase cysteinyl leukotrienes levels that may induce angioedema. This side effect is less common with COX2 inhibitors than with COX1 inhibitors.

However, histaminergic angioedema may sometimes occur spontaneously, and the possible cause cannot always be determined. ${ }^{8,14}$ Idiopathic histaminergic AAE (IH-AAE) may be diagnosed when any possible causes of histaminergic angioedema are excluded (foods, drugs, animal dander, aeroallergens, insects stings, latex, and others), and the symptoms respond well to antihistamine treatment (Figure 1).

Recurrent angioedema and/or urticaria attacks can last for months, sometimes years. Angioedema occurs in 50\% of patients with chronic spontaneous urticaria. A total of $10 \%$ of patients with this condition may have isolated angioedema without urticaria. ${ }^{1,-5}$ In the diagnosis of IH-AAE, allergens, drugs, other acquired forms, as well as HAE must be ruled out. In IH-AAE, recurrent angioedema attacks occur without any identified external stimulus. The face and extremities are most commonly affected, with swelling lasting for hours to days. Laryngeal involvement is rare. Chronic urticaria/ angioedema lasts 3-5 years in most patients, although it is known that some cases can last for decades. ${ }^{3,4}$ In $30 \%$ of these patients, COX1-inhibiting drugs may trigger urticaria or angioedema. In cases where recurrent angioedema is isolated and not accompanied by urticaria, response to antihistamines supports IH-AAE diagnosis. ${ }^{47}$ However, patients do not always respond to antihistamine therapy. No reduction in attack frequency was reported in $36 \%$ of patients with IH-AAE who received prophylactic antihistamines, and no exacerbation was reported in another $5 \% .^{52}$ This high rate of nonresponse to antihistamine therapy may lead to further confusion during diagnosis.

\section{Idiopathic Nonhistaminergic Acquired Angioedema}

Angioedema may also occur in the absence of mast cell activation through mediators other than histamine. A lack of itching or urticaria and nonresponse to antihistamines should raise the suspicion of this type of angioedema, classified as nonhistaminergic angioede- ma. The primary mechanisms of nonhistaminergic angioedema are drug-induced changes in the kinin pathway and some complement system disorders. In this condition, angioedema might occur owing to mediators' formation, such as bradykinin. ${ }^{16,47}$ It is known that a significant portion, if not all, of nonhistaminergic angioedema is mediated by bradykinin. Bradykinin is a nonapeptide that is released from high-molecular-weight kininogen by kallikrein and plays a role in the control of vascular tone. Kallikrein is also activated through the contact system. Bradykinin binds to transmembrane B2Rs in the vascular endothelium. Typical examples of nonhistaminergic angioedema are ACEi-induced, hereditary, and acquired angioedema. ${ }^{47}$ The use of dipeptidyl peptidase-IV (DPPIV) inhibitor and chronic neprilysin inhibition are also associated with an increased risk of nonhistaminergic angioedema. ${ }^{53,54}$ This angioedema does not differ in appearance from histaminergic angioedema. However, some features facilitate the differentiation of bradykinin-mediated angioedema from histaminergic angioedema (Table 1). Bradykinin-mediated angioedema is not accompanied by urticaria. The angioedema takes longer to develop and to resolve. Upper respiratory tract involvement, including in the larynx, can be deadly, and edema in the intestinal walls can cause severe abdominal pain attacks that are challenging to distinguish from an acute abdomen. Because bradykinin is the mediator responsible for increased permeability in most of the cases, antihistamines, corticosteroids, and adrenaline are ineffective.

Idiopathic nonhistaminergic AAE (InH-AAE) should be considered when all the other types of recurrent angioedema (including drugs, hereditary, and acquired angioedema) have been ruled out and patients do not respond to high-dose antihistamines. ${ }^{47}$ In InHAAE, the average age at symptom onset is 36 years, and $56 \%$ of patients have more than 12 angioedema attacks per year. ${ }^{55}$ Attacks are most common in the face and extremities. The upper respiratory tract can be affected, and approximately $20 \%$ of cases may have abdominal and genital attacks. ${ }^{55}$ There is limited information that bradykinin plays a role in the pathophysiology of this disease. ${ }^{56}$ Some of these patients respond well to B2R antagonists. ${ }^{57}$ However, the response shown by some patients to anti-IgE therapy, such as omalizumab, suggests that a more complex mechanism might underlie this type of angioedema. ${ }^{58}$

\section{Acquired Angioedema Related to Angiotensin-Converting Enzyme Inhibitors}

Angioedema is seen in only $0.3-0.68 \%$ of patients taking ACEis. ${ }^{59}$ However, because ACEis are one of the most commonly prescribed drugs worldwide, angioedema is estimated widespread, with at least a 3-fold greater risk in African Americans. ${ }^{11}$ Approximately half of the patients admitted to the hospital for acute angioedema are patients receiving ACEi therapy. ${ }^{11,12}$ The prevalence of ACEi-induced angioedema (ACEi-AE) varies from one country to another, depending on the country-specific percentage of prescription of ACEIs, and ranges from 0.4-2.6 per 10,000 people. ${ }^{60}$

$\mathrm{ACE}$ is involved in the conversion of angiotensin I to angiotensin II. ACE, also known as kininase II, is also responsible for the degradation of bradykinin. Therefore, ACEis may cause bradykinin-mediated angioedema in a small percentage of users. ${ }^{11}$ Patients 
presenting with ACEi-AE were shown to have increased bradykinin concentrations owing to reduced degradation. ${ }^{61}$

A genetic cause has not been identified for ACEi-AE, but increased risk has been associated with polymorphisms in the XPEPNP2, $B D K R B 2-9 /+9$, and $N E P$ genes. ${ }^{62}$ Although it represents a rare adverse effect for ACEis $(<1 \%)$, these commonly used drugs are the cause of a significant percentage of emergency presentations due to isolated angioedema. ${ }^{12}$ It is more common in African Americans and patients with HAE. In some cases, HAE that has remained latent for many years may manifest after initiating an ACEI. Some studies have reported a higher prevalence in people aged $>65$ years, smokers, women, transplant recipients, and users of immunosuppressive drugs. The prevalence is lower in people with diabetes. ${ }^{18,63}$

Angioedema typically occurs in the lips, tongue, mouth, pharynx, and subglottic regions. Although death is uncommon, patients may require hospitalization and intensive care monitoring owing to airway involvement. There is no urticaria or itching. Intestinal edema is another clinical presentation caused by this group of drugs. It may cause sudden-onset abdominal pain, diarrhea, and vomiting. The clinical onset of angioedema occurs within a week after initiating the medication in $>50 \%$ of cases. ${ }^{11,12}$ It can also appear after the first 6 months in $>25 \%$ of cases and years later in rare cases. The angioedema attacks often end after ACEi cessation, but in 25\% of patients, they may persist for some time despite discontinuing treatment. ${ }^{61,63}$ If angioedema occurs at any stage of ACEi therapy, the drug should be discontinued immediately. Substitution with a different agent of the same pharmaceutical class should be considered as a contraindication. Angiotensin II receptor blockers provide similar benefits to ACEis without affecting bradykinin metabolism. In patients who develop angioedema when taking ACEis, the risk of angioedema when switching therapy to angiotensin II receptor blockers is between $2 \%$ and $17 \%{ }^{64}$

There is no standard laboratory test used to diagnose ACEi-AE, which is usually based on clinical findings. Because the primary mechanism in ACEi-AE is through bradykinin, treatment with antihistamines, corticosteroids, and epinephrine is usually ineffective. Instead, better efficacy could be expected from drugs used to treat acute attacks in HAE, such as fresh-frozen plasma, C1-INH concentrate, bradykinin receptor antagonists, or kallikrein inhibitors. ${ }^{65}$ Although the B2R antagonist icatibant was previously shown to be effective in ACEi-AE, a meta-analysis found that this drug was not superior to a placebo. ${ }^{66,67}$ However, because the patient selection criteria, the primary endpoints of the studies, and the time elapsed between the onset of symptoms and treatment administration differ in all these studies, these results should be evaluated carefully.

\section{Acquired Angioedema with C1-Inhibitor Deficiency} AAE with C1-INH deficiency (C1-INH-AAE) (ORPHA:528663) is an extremely rare disease. The estimated prevalence may vary between 1 in 100,000 and 1 in 500,000. ${ }^{21}$ The prevalence of C1INH-AAE in Denmark is estimated to be 1.5 in 1 million. ${ }^{68}$ However, the actual prevalence might be higher because this disease often remains unrecognized. Fewer than 1 in 10 patients with C1-INH deficiency have C1-INH-AAE. ${ }^{68,69}$ Around 200 cases have been de- scribed in the literature ${ }^{69}$ The clinical presentation is very similar to that of HAE, making it difficult to distinguish these 2 conditions clinically. Recurrent angioedema attacks are not accompanied by urticaria and typically resolve in 2-5 days. Unlike HAE, it is not associated with genetic mutations; therefore, there is no family history. Symptoms tend to appear later in life, usually after the age of 40 years. ${ }^{69}$

C1-INH-AAE is most commonly associated with B-cell lymphoproliferative diseases and autoimmune diseases. ${ }^{70}$ The lymphoproliferative disease can range from the production of an unknown monoclonal antibody (monoclonal gammopathy of unknown significance [MGUS]) to B-cell lymphomas. Some patients with AAE have autoantibodies against C1-INH. Traditionally, C1INH-AAE is subclassified as type I (associated with lymphoproliferative diseases and excessive $\mathrm{C} 1-\mathrm{INH}$ consumption) and type II (develops secondary to the production of anti-C1INH autoantibodies and is often associated with MGUS).$^{70}$ However, the simultaneous availability of autoantibodies to $\mathrm{C} 1-\mathrm{INH}$ together with the presence of lymphoproliferative disease leading to excessive consumption of $\mathrm{C} 1-\mathrm{INH}$ suggests that this distinction is actually fictitious. ${ }^{69,70}$

In approximately $40 \%$ of patients, angioedema attacks begin 1 year before the underlying disease is diagnosed. $\mathrm{C} 4$ and $\mathrm{C} 1-\mathrm{INH}$ levels and $\mathrm{C} 1$ function are below the reference levels. C1q concentrations are low in most patients with $\mathrm{C} 1-\mathrm{INH}-\mathrm{AAE}$ and generally normal in patients with HAE, but patients who do not conform to these patterns have also been described. ${ }^{33,70}$ Although treating the underlying disease responsible for the development of AAE effectively controls angioedema attacks, treatment response varies. ${ }^{71}$ Treatment options are the same as in HAE. Anti-CD20 monoclonal antibody therapy to reduce $\mathrm{B}$ cells was reported to be effective in patients with $\mathrm{C} 1-\mathrm{INH}-\mathrm{AAE}{ }^{71}$

\section{OTHER RARE CAUSES OF RECURRENT ANGIOEDEMA WITHOUT WHEALS}

Apart from the diseases mentioned in this article, there are also some medical conditions that may cause recurrent angioedema. A total of $15 \%$ of cases with hypereosinophilic syndrome may experience angioedema. ${ }^{72}$ It may occur owing to the release of vasodilator mediators from eosinophils or the activation of cutaneous mast cells by other eosinophil-derived mediators. ${ }^{72}$ Other conditions that may cause angioedema include episodic angioedema with eosinophilia (Gleich syndrome), which is rare and classified as cytokine-associated angioedema; nonepisodic angioedema with eosinophilia; systemic capillary leak syndrome (Clarkson syndrome); and nodules, eosinophilia, rheumatism, dermatitis, and swelling syndrome. ${ }^{73,74}$ Viral infections are among the common causes of urticaria in children but may also cause isolated angioedema. Parvovirus B19 is known to cause angioedema in newborns. ${ }^{75}$

\section{DIFFERENTIAL DIAGNOSIS}

There are several conditions referred to as pseudoangioedemas that can be confused with angioedema and result in referrals to allergy or dermatology clinics. The diseases that should be considered in the differential diagnosis of angioedema are contact dermati- 
TABLE 2. Laboratory findings in different types of angioedema without wheals

\begin{tabular}{|c|c|c|c|c|}
\hline Angioedema & $\mathrm{C} 4$ & $\mathrm{C} 1-\mathrm{INH}$ & C1-INH function & $\mathrm{C} 1 \mathrm{q}$ \\
\hline C1-INH-AAE type I & Low & Low $(<50)$ & $<50 \%$ & Normal \\
\hline C1-INH-AAE type II & Low & Normal/increased & $<50 \%$ & Normal \\
\hline nC1-INH-HAE & Normal & Normal & Normal & Normal \\
\hline C1-INH-AAE & Low & Low/normal & $<50 \%$ & Low/normal \\
\hline ACEi-AE & Normal & Normal & Normal & Normal \\
\hline IH-AAE/InH-AAE & Normal & Normal & Normal & Normal \\
\hline Allergic & Normal & Normal & Normal & Normal \\
\hline NSAID dependent & Normal & Normal & Normal & Normal \\
\hline
\end{tabular}

ACEi-AE, angiotensin-converting enzyme inhibitor-induced acquired angioedema; C1-INH-AAE, acquired angioedema with C1 inhibitor deficiency; CI-INH, C1 inhibitor; C1-INH$\mathrm{HAE}$, hereditary angioedema with $\mathrm{C} 1$ inhibitor; $\mathrm{nC} 1-\mathrm{INH}-\mathrm{HAE}$, hereditary angioedema with normal $\mathrm{C} 1$ inhibitor; IH-AAE, idiopathic histaminergic acquired angioedema; InH-AAE, idiopathic nonhistaminergic acquired angioedema; NSAID, nonsteroidal anti-inflammatory drug.

tis, cellulitis, Morbihan's disease, systemic lupus erythematosus, scleroderma, dermatomyositis, Sjögren's syndrome, DRESS (drug reaction with eosinophilia and systemic symptoms) syndrome, hypocomplemantemic urticarial vasculitis, myxedema, vena cava superior syndrome, subcutaneous emphysema, Clarkson's disease, orofacial granulomatosis, blepharochalasis, cluster headache, Ascher syndrome, and dermatitis artefacta. ${ }^{76-78}$

\section{EXPERT COMMENTARY and CONCLUSION}

The most important step in the evaluation of patients with recurrent angioedema without wheals is taking their personal and family history. Symmetrical swelling points to other etiologies, whereas laryngeal edema should suggest histamine- or bradykinin-mediated angioedema. The clinician must first ascertain whether the patient has urticaria, rash, itching, bronchospasm, presyncope, or hypotension because the presence of any of these findings will narrow the assessment to histaminergic events, including anaphylaxis. For acute angioedema accompanied by these symptoms, there is usually an external trigger, such as foods, drugs, physical contact, or insect bites/stings. Ocular swelling that occurs with rhinitis and conjunctivitis is usually caused by aeroallergens. Apart from the presence of other allergic symptoms, angioedema that appears more rapidly, improves in a shorter time, and responds to treatments used in allergic conditions also suggests histaminergic angioedema (Table 1). Laboratory examinations performed during a severe angioedema attack may be rarely helpful in identifying a mast cell-mediated event. Although high serum tryptase concentration supports a diagnosis of anaphylaxis, normal values are not enough to rule out anaphylaxis.

The presence of urticaria usually excludes the diagnoses of bradykinin-mediated hereditary, acquired and ACEi-induced, DPP-IV inhibitor-induced, and neprilysin inhibitor-induced angioedema. In patients with recurrent angioedema attacks without urticaria, generalized pruritus, rash, or hypotension, an in-depth examination should specifically encompass ACEis, angiotensin II antagonists, and NSAIDs. The first appearance of angioedema attacks may occur 6 months or even years after the initiation of ACEi in 25\% of ACEi-AE cases. This fact might make diagnosis challenging. Again, although these patients take ACEis every day, it is an inter- esting issue why the attacks are intermittent. Another interesting point in this context is that in $25 \%$ of cases, angioedema attacks can persist for weeks or months after drug discontinuation. C1INH-HAE, which may remain asymptomatic for years in some patients, may lead to the first angioedema attack after the initiation of ACEis. Again, the age of starting antihypertensive drugs may be similar to the age of onset of angioedema attacks in patients with C1-INH-HAE. Therefore, it may be necessary to distinguish whether the angioedema is caused by ACEi, C1-INH-HAE, or C1INH-AAE. HAE should be considered in cases of recurrent angioedema dating back to childhood or adolescence and laryngeal edema accompanied by attacks of abdominal pain, especially if there is a positive family history. However, it should be kept in mind that $6-25 \%$ of patients with C1-INH-HAE may not always have symptomatic patients in their family. It is interesting that $5-10 \%$ of patients with C1-INH-HAE do not have a mutation in the SERPING1 gene and that $5 \%$ of those with a mutation in this gene region have no symptoms. Measuring the concentrations and function of complement components $\mathrm{C} 4$ and $\mathrm{C} 1-\mathrm{INH}$ are recommended as initial tests for patients with suspected HAE or AAE. If the patient is middle aged at the time of symptom onset and/or has no family history, $\mathrm{C} 1 \mathrm{q}$ concentration should also be evaluated to identify C1-INH-HAE (Table 2). It should be good to bear in mind that angioedema attacks might start 1 year before the underlying disease in $40 \%$ of C1-INH-HAE cases. The lack of a standard biochemical laboratory test for patients with IH-AAE, InH-AAE, ACEi-AE, and nC1-INH-HAE yet makes the diagnosis more difficult. Especially in patients without a family history or in patients without FXII, ANGPT-1, KNG-1, PLG, MYOF mutations, it can be very challenging to diagnose.

Future efforts should focus on increasing the awareness of all rare types of angioedema among physicians and developing more straightforward and more accessible diagnostic methods.

Conflict of Interest: The author has no conflicts of interest to declare.

Funding: The author declared that this study has received no financial support.

\section{REFERENCES}

1. Saini SS. Urticaria and angioedema in Middleton's Allergy Principles and Practice. ed Adkinson NF, et al. 8th edition; Philadelphia 2014;575-587. [Crossref] 
2. Frigas E, Nzeako UC. Angioedema: pathogenesis, differential diagnosis, and treatment. Clin Rev Allergy Immunol. 2002;23(2):217-231. [Crossref]

3. Greaves MW. Chronic urticaria. N Engl J Med. 1995;332(26):1767-1772. [Crossref]

4. Kaplan AP. Chronic urticaria: pathogenesis and treatment. J Allergy Clin Immunol. 2004;114(3):465-474. [Crossref]

5. Sabroe RA, Seed PT, Francis DM, Barr RM, Black AK, Greawes MW. Chronic idiopathic urticaria: comparison of the clinical features of patients with and without anti-Fc epsilon RI or anti-IgE autoantibodies. J Am Acad Dermatol. 1999;40(3):443450. [Crossref]

6. Rasmussen EHR, Bindslev-Jensen C, Bygum A. Angioedema-assessment and treatment. Tidsskr Nor Laegeforen. 2012;132(2):2391-2395. [Crossref]

7. Lin RY, Shah SN. Increasing hospitalizations due to angioedema in the United States. Ann Allergy Asthma Immunol. 2008;101(2):185-92. [Crossref]

8. Madsen F, Altermann J, Linneberg A. Epidemiology of non-hereditary angioedema. Acta Derm Venerol. 2012;92(5):475-479. [Crossref]

9. Zingale LC, Beltrami L, Zanichelli A, et al. Angioedema without urticaria: a large clinical survey. CMAJ. 2006;175(9):1065-1070. [Crossref]

10. Tai S, Mascaro M, Goldstein NA. Angioedema: a review of 367 episodes presenting to three tertiary care hospitals. Ann Otol Rhinol Laryngol. 2010;119(12):836-841. [Crossref]

11. Kostis JB, Kim HJ, Rusnak J, et al. Incidence and characteristics of angioedema associated with enalapril. Arch Intern Med. 2005;165(14):1637-1642. [Crossref]

12. Yde Holm JP, Ovesen T. Increasing rate of angiotensin-converting enzyme inhibitor-related upper airway angio-oedema. Dan Med J. 2012;59(6):A4449.

13. Romano A, Viola M, Guéant-Rodriguez RM, Gaeta F, Pettinato R, Guéant JL. Imipenem in patients with immediate hypersensitivity to penicillins. $N$ Engl J Med. 2006;354(26):2835-2837. [Crossref]

14. Grigoriadou S, Longhurst HJ. Clinical Immunology Review Series: An approach to the patient with angio-oedema. Clin Exp Immunol. 2009;155(3):367-377. [Crossref]

15. Cicardi M, Aberer W, Banerji A, et al. Classification, diagnosis, and approach to treatment for angioedema: consensus report from the Hereditary Angioedema International Working Group. Allergy. 2014;69(5):602-616. [Crossref]

16. Temiño VM, Peebles RS. The spectrum and treatment of angioedema. Am J Med. 2008;121(4):282-286. [Crossref]

17. Bernstein IL. Hereditary angioedema: a current state-of-the-art review, II: historical perspective of non-histamine-induced angioedema. Ann Allergy Asthma Immunol. 2008;100(Suppl 2):S2-S6. [Crossref]

18. Zuraw BL, Christiansen SC. Hereditary angioedema and bradykinin mediated angioedema. in Middleton's Allergy Principles and Practice. ed Adkinson NF et al. 8th edition; Philadelphia 2014; 588-600. [Crossref]

19. Nzeako UC, Frigas E, Tremaine WJ. Hereditary angioedema: a broad review for clinicians. Arch Intern Med. 2001;161(20):2417-2429. [Crossref]

20. Zuraw BL. Hereditary angioedema. N Engl J Med. 2008;359(10):1027-1036. [Crossref]

21. Winnewisser J, Rossi M, Späth P, Bürgi H. Type I hereditary angioedema: variability of clinical presentation and course within two large kindreds. J Intern Med. 1997;241(1):39-46. [Crossref]

22. Bork K, Meng G, Staubach P, Hardt J. Hereditary angioedema: new findings concerning symptoms, affected organs, and course. Am J Med. 2006;119(3):267-274. [Crossref]

23. Nussberger J, Cugno M, Cicardi M, Agostoni A. Local bradykinin generation in hereditary angioedema. J Allergy Clin Immunol. 1999;104(6):1321-1322. [Crossref]

24. Agostoni A, Cicardi M. Hereditary and acquired C1-inhibitor deficiency: biological and clinical characteristics in 235 patients. Medicine (Baltimore). 1992;71(4):206215. [Crossref]

25. Frank MM. Hereditary angioedema: the clinical syndrome and its management in the United States. Immunol Allergy Clin North Am. 2006;26(4):653-668. [Crossref]

26. Agostoni A, Cicardi M, Cugno M, Zingale LC, Gioffré D, Nussberger J. Angioedema due to angiotensin-converting enzyme inhibitors. Immunopharmacol. 1999;44(12):21-25. [Crossref]

27. Frank MM. Effect of sex hormones on the complement-related clinical disorder of hereditary angioedema. Arthritis Rheum. 1979;22(11):1295-1299. [Crossref]

28. Bouillet L, Longhurst H, Boccon-Gibod I, et al. Disease expression in women with hereditary angioedema. Am J Obstet Gynecol. 2008;199(5):484.e1-4. doi: 10.1016/j. ajog.2008.04.034. Epub 2008 Jun 13. [Crossref]

29. Bouillet L. Hereditary angioedema in women. Allergy Asthma Clin Immunol. 2010;6(1):17. [Crossref]
30. Hofman ZLM, Relan A, Hack CE. Hereditary angioedema attacks: local swelling at multiple sites. Clin Rev Allergy Immunol. 2016;50(1):34-40. [Crossref]

31. Talavera A, Larraona JI, Ramos JI, et al. Hereditary angioedema: an infrequent cause of abdominal pain with ascites. Am J Gastroenterol. 1995;90:471-474.

32. Lumry WR, Castaldo AJ, Vernon MK, et al. The humanistic burden of hereditary angioedema: Impact on health-related quality of life, productivity, and depression. Allergy Asthma Proc. 2010;31(5):407-414. [Crossref]

33. Gökmen NM, Gülbahar O, Onay H, et al. Deletions in SERPING1 lead to lower $\mathrm{Cl}$ inhibitor function: lower $\mathrm{C} 1$ inhibitor function can predict disease severity. Int Arch Allergy Immunol. 2019;178(1):50-59. [Crossref]

34. Bork K, Hardt J, Schicketanz KH, Ressel N. Clinical studies of sudden upper airway obstruction in patients with hereditary angioedema due to $\mathrm{C} 1$ esterase inhibitor deficiency. Arch Intern Med. 2003;163(10):1229-1235. [Crossref]

35. Bork K, Hardt J, Witzke G. Fatal laryngeal attacks and mortality in hereditary angioedema due to C1-INH deficiency. J Allergy Clin Immunol. 2012;130(3):692-697. [Crossref]

36. Bowen T, Cicardi M, Bork K, et al. Hereditary angioedema: a current state-of-the art review, VII: Canadian Hungarian 2007 International Consensus Algorithm for the diagnosis, therapy, and management of Hereditary angioedema. Ann Allergy Asthma Immunol. 2008;100(Suppl 2):S30-S40. [Crossref]

37. Cicardi M, Agostoni A. Hereditary angioedema. $N$ Engl J Med. 1996;334(25):16661667. [Crossref]

38. Stenson PD, Mort M, Ball EV, et al. The Human Gene Mutation Database: towards a comprehensive repository of inherited mutation data for medical research, genetic diagnosis and next-generation sequencing studies. Hum Genet. 2017;136(6):665-677. [Crossref]

39. Ponard D, Gaboriaud C, Charignon D, et al. SERPING1 mutation update: mutation spectrum and $\mathrm{C} 1$ inhibitor phenotypes. Hum Mutat. 2020;41(1):38-57. [Crossref]

40. Tarzi MD, Hickey A, Forster T, Mohammadi M, Longhurst HJ. An evaluation of tests used for the diagnosis and monitoring of $\mathrm{C} 1$ inhibitor deficiency: normal serum $\mathrm{C} 4$ does not exclude hereditary angioedema. Clin Exp Immunol. 2007;149(3):513-516. [Crossref]

41. Bork K, Bamstedt SE, Koch P, Traupe H. Hereditary angioedema with normal C1-inhibitor activity in women. Lancet. 2000;356:213-217. [Crossref]

42. Bork K, Gul D, Dewald G. Hereditary angioedema with normal $\mathrm{C} 1$ inhibitor in a family with affected women and men. Br J Dermatol. 2006;154:542-545. [Crossref]

43. Bork K. Hereditary angioedema with normal $\mathrm{C} 1$ inhibitor activity including hereditary angioedema with coagulation factor XII gene mutations. Immunol Allergy Clin North Am. 2006;26(4):709-724. [Crossref]

44. Bouillet L, Boccon-Gibod I, Launay D, et al. Hereditary angioedema with normal C1 inhibitor in a French cohort: Clinical characteristics and response to treatment with icatibant. Immun Inflamm Dis. 2017;5(1):29-36. [Crossref]

45. Bork K, Machnig T, Wulff K, Witzke G, Prusty S, Hardt J. Clinical features of genetically characterized types of hereditary angioedema with normal $\mathrm{Cl}$ inhibitor: a systematic review of qualitative evidence. Orphanet J Rare Dis. 2020;15(1):289. [Crossref]

46. Ariano A, D'Apolito M, Bova M, et al. A myoferlin gain-of-function variant associates with a new type of hereditary angioedema. Allergy. 2020;75(11):2989-2992. [Crossref]

47. Veronez CL, Grumach AS. Angioedema without urticaria: novel findings which must be measured in clinical setting. Curr Opin Allergy Clin Immunol. 2020;20(3):253260. [Crossref]

48. Bork K, Wulff K, Steinmüller-Magin L, et al. Hereditary angioedema with a mutation in the plasminogen gene. Allergy. 2018;73(2):442-450. [Crossref]

49. Gökmen NM, Gülbahar O, Koç ZP, et al. How familiar are internists with a potentially deadly orphan disease? Hereditary angioedema. Turkiye Klinikleri J Med Sci. 2015;35(2):67-72. [Crossref]

50. Zanichelli A, Magerl M, Longhurst H, Fabient V, Maurer M. Hereditary angioedema with $\mathrm{C} 1$ inhibitor deficiency: delay in diagnosis in Europe. Allergy Asthma Clin Immunol. 2013;9(1):29. [Crossref]

51. Gülbahar O, Gökmen NM. Herediter Anjioödem. Ege Üniversitesi Basımevi, İzmir; 2014.

52. Hofman ZLM, van West N, Hack CE, Knulst AC, Maas C, Röckmann H. High occurrence of antihistamine resistance in patients with recurrent idiopathic angioedema. Clin Transl Allergy. 2019;9, 35. [Crossref]

53. Brown NJ, Byiers S, Carr D, Maldonado M, Warner BA. Dipeptidyl peptidase-IV inhibitor use associated with increased risk of ACE inhibitor-associated angioedema. Hypertension. 2009;54(3):516-523. [Crossref] 
54. Campbell DJ. Long-term neprilysin inhibition - implications for ARNIs. Nat Rev Cardiol. 2017;14(3):171-186. [Crossref]

55. Andrasi N, Vezseli N, Kohalmi KV, et al. Idiopathic nonhistaminergic acquired angioedema versus hereditary angioedema. $J$ Allergy Clin Immunol Pract. 2018;6(4):1205-1208. [Crossref]

56. Cugno M, Tedeshi A, Nussberger J. Bradykinin in idiopathic non-histaminergic angioedema. Clin Exp Allergy. 2017;47(1):139-140. [Crossref]

57. Colas C, Montoiro R, Fraj J, Garcés M, Cubero JL, Caballero T. Non-histaminergic idiopathic angioedema: clinical response to icatibant. J Invest Allergol Clin Immunol. 2012;22(7):520-521.

58. Brunetta E, Shiffer D, Folci M, et al. Omalizumab for idiopathic nonhistaminergic angioedema: evidence for efficacy in 2 patients. Case Reports Immunol. 2018;2018:8067610. [Crossref]

59. Bezalel S, Mahlab-Guri K, Asher I, Werner B, Sthoeger ZM. Angiotensin-converting enzyme inhibitor-induced angioedema. Am J Med. 2015;128(2):120-125. [Crossref]

60. Aygören-Pürsün E, Magerl M, Maetzel A, Maurer M. Epidemiology of Bradykinin-mediated angioedema: a systematic investigation of epidemiological studies. Orphanet J Rare Dis 2018;13(1):73. [Crossref]

61. Hubers SA, Kohm K, Wei S, et al. Endogenous bradykinin and B1-B5 during angiotensin-converting enzyme inhibitor-associated angioedema. J Allergy Clin Immunol. 2018;142(5):1636-1639.e5. [Crossref]

62. Ali HA, Lomholt AF, Mahmoudpour SH, et al. Genetic susceptibility to angiotensin-converting enzyme-inhibitor induced angioedema: a systematic review and evaluation of methodological approaches. PloS One. 2019;14(11):e0224858. [Crossref]

63. Beltrami L, Zanichelli A, Zingale L, Vacchini R, Carugo S, Cicardi M. Long-term follow-up of 111 patients with angiotensin-converting enzyme inhibitor-related angioedema. J Hypertens. 2011; 29(11):2273-2277. [Crossref]

64. Haymore BR, Yoon J, Mikita CP, Klote MM, De Zee KJ. Risk of angioedema with angiotensin receptor blockers in patients with prior angioedema associated with angiotensin-converting enzyme inhibitors: a meta-analysis. Ann Allergy Asthma Immunol. 2008;101(5):495-499. [Crossref]

65. Riha HM, Summers BB, Rivera JV, Van Berkel MA. Novel therapies for angiotensin-converting enzyme inhibitor-induced angioedema: a systematic review of current evidence. J Emer Med. 2017; 53(5):662-679. [Crossref]
66. Baş M, Greve J, Stelter K, et al. A randomized trial of icatibant in ACE-inhibitor-induced angioedema. $N$ Engl J Med. 2015;372(5):418-425. [Crossref]

67. Jeon J, Lee YJ, Lee SY. Effect of icatibant on angiotensin-converting enzyme inhibitor-induced angioedema: a meta-analysis of randomized controlled trials. J Clin Pharm Ther. 2019;44(5):685-692. [Crossref]

68. Bygum A, Vestergaard H. Acquired angioedema occurrence, clinical features and associated disorders in a Danish nationwide patient cohort. Int Arch Allergy Immunol. 2013;162(2):149-155. [Crossref]

69. Kivity S. New perspectives in acquired angioedema. Isr Med Assoc J. 2014;16(5):313-314.

70. Castelli R, Zanichelli A, Cicardi M, Cugno M. Acquired C1-inhibitor deficiency and lympho- proliferative disorders: a tight relationship. Crit Rev Oncol Hematol. 2013;87(3):323-332. [Crossref]

71. Dreyfus DH, Rim Na C, Randolph CC, Kearney D, Price C, Podell D. Successful rituximab B lymphocyte depletion therapy for angioedema due to acquired $\mathrm{C} 1$ inhibitor protein deficiency: association with reduced $\mathrm{C} 1$ inhibitor protein autoantibody titers. Isr Med Assoc J. 2014;16(5):315-316.

72. Plötz SG, Hüttig B, AignerB, et al. Clinical overview of cutaneous features in hypereosinophilic syndrome. Curr Allergy Asthma Rep. 2012;12(2):85-98. [Crossref]

73. Banerji A, Weller PF, Sheikh J. Cytokine-associated angioedema syndromes including episodic angioedema syndromes with eosinophilia (Gleich's Syndrome). Immunol Allergy Clin North Am. 2006;26(4):769-781. [Crossref]

74. Tahirkheli NK, Greipp PR. Treatment of systemic capillary leak syndrome with terbutaline and theophylline. A case series. Ann Intern Med. 1999;130(11):905-909. [Crossref]

75. Miyagawa S, Takahashi Y, Nagai A, et al. Angiooedema in a neonate with IgG antibodies to parvovirus B19 following intrauterine parvovirus B19 infection. Br J Dermatol. 2000;143(2):428-430. [Crossref]

76. Kaplan AP, Greaves MW. Angioedema. J Am Acad Dermatol. 2005;53(3):373-388. [Crossref]

77. Frigas E, Park M. Idiopathic recurrent angioedema. Immunol Allergy Clin North Am. 2006;26(4):739-751. [Crossref]

78. Andersena MF, Longhurstd HJ, Rasmussenb ER, Bygum A. How not to be misled by disorders mimicking angioedema: a review of pseudoangioedema. Int Arch Allergy Immunol. 2016;169(3):163-170. [Crossref] 